\title{
Comment on 'The efficacy and toxicity of gemcitabine, carboplatin and bevacizumab in metastatic breast cancer'
}

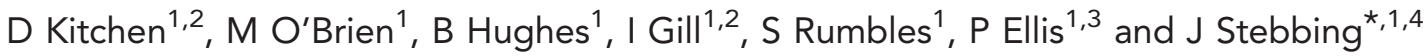

${ }^{1}$ Leaders in Oncology Care (the LOC), 95 Harley Street, London W1G 6AF, UK; ${ }^{2}$ Barts and The London School of Medicine and Dentistry, Garrod Building, Turner Street, London E1 2AD, UK; ${ }^{3}$ Guys and St Thomas NHS Foundation Trust, Cancer Management Office, 4th Floor, Bermondsey Wing, Guy's Hospital, Great Maze Pond, London SE1 9RT, UK and ${ }^{4}$ Department of Medical Oncology, Imperial College Healthcare NHS Trust, Charing Cross Hospital, 1st Floor, E Wing, Fulham Palace Road, London W6 8RF, UK

Sir,

As pointed out in the excellent article by Miles et al (2013), the identification of patients benefiting most from bevacizumab remains elusive. The activity of the gemcitabine and platinum combination in a variety of neoplasms is well described. In metastatic breast cancer, it has often been used as a standard regimen in patients with triple-negative disease, despite a lack of comparative phase 3 data, especially in anthracycline and taxane pre-treated individuals (Perez, 2004; Heinemann et al, 2006; Sanchez-Escribano Morcuende et al, 2007; Maisano et al, 2011). The role of gemcitabine and carboplatin in this setting has been highlighted by its use in the control arm in randomised trials of PARP inhibitors (Foulkes et al, 2010; Stebbing et al, 2010; O’Shaughnessy, 2012).

The use of bevacizumab in breast cancer has been associated with controversy and it has been combined with a wide variety of cytotoxics (Jones and Ellis, 2011), some combinations thought to have more benefits than others (Miller et al, 2007; O'Shaughnessy and Brufsky, 2008; Brufsky et al, 2011; Hamilton and Blackwell, 2011; Brufsky et al, 2012; Kesikli and Kilickap, 2012). To the best of our knowledge, the activity and toxicity of gemcitabine, carboplatin and bevacizumab has not been described in women with metastatic breast cancer.

The oncology cohort at the Leaders in Oncology Care (LOC) clinic in London includes over 11000 patients with data prospectively recorded for the period between May 2005 and September 2011, as previously described (Kitchen et al, 2012). When patients attend the clinic for treatment, their toxicities are recorded electronically on the MOSAIQ electronic medical records package (Elekta, Stockholm, Sweden) using the Chemotherapy
Toxicity Criteria (CTCAE3.0) scale. We identified during this time, any patients with metastatic breast cancer regardless of histology, who received treatment with gemcitabine, carboplatin and bevacizumab.

Patients received bevacizumab $\left(15 \mathrm{mgkg}^{-1}\right.$ on day 1$)$, gemcitabine $\left(1000 \mathrm{mg} / \mathrm{m}^{2}\right.$ on days 1 and 8$)$ and carboplatin (AUC 5 on day 1) for up to eight 3 weekly cycles. A total of 14 patients were identified who received this combination; the median progression-free survival (PFS) measured 5.1 months (95\% CI 2.2-8.0 months) with a median overall survival (OS) of 8.3 months (95\% CI 6.5-10.0 months); $71 \%$ were alive at 6 months. Their median age was 45 years old (range 31-66 years). A total of 10 patients $(71 \%)$ in the cohort had triple-negative breast cancer, with the entire cohort being negative for HER-2. The liver, lung and bone metastases were the commonest metastatic sites, with six individuals (43\%) having metastases at all these locations. The median number of treatments received for metastatic disease prior to commencing treatment with gemcitabine, carboplatin and bevacizumab was 2 (range $0-4$ ), with nine patients (64\%) having received either an anthracycline, taxane or both in their previous metastatic regimens prior to receiving therapy; gemcitabine, carboplatin and bevacizumab was first-line treatment in three individuals (21\%).

The most common grade III/IV toxicities (Table 1) were fatigue in six patients $(43 \%)$ and pain in five patients, though this latter side effect was probably related to the cancer itself (36\%). Alopecia and bleeding were not reported. Figure 1 demonstrates the OS and PFS curves for this single cohort.

The median number of cycles of gemcitabine, carboplatin and bevacizumab administered was 5 (range 1-8). An objective partial 


\section{Table 1. Grade III/IV toxicities}

\begin{tabular}{|l|c|}
\hline Side effects & $\mathbf{N}(\%)$ \\
\hline Fatigue & $6(43)$ \\
\hline Diarrhoea & $2(14)$ \\
\hline Pain & $5(36)$ \\
\hline Dry skin & $1(7)$ \\
\hline Nausea & $1(7)$ \\
\hline Vomiting & $1(7)$ \\
\hline Severe anaphylaxis & $1(7)$ \\
\hline Hand-foot reaction & $1(7)$ \\
\hline Cough & $1(7)$ \\
\hline Insomnia & $2(14)$ \\
\hline Mucositis & $1(7)$ \\
\hline Hot flushes & $1(7)$ \\
\hline Nerve pain & $1(7)$ \\
\hline Hypertension & $1(7)$ \\
\hline Taste changes & $1(7)$ \\
\hline
\end{tabular}

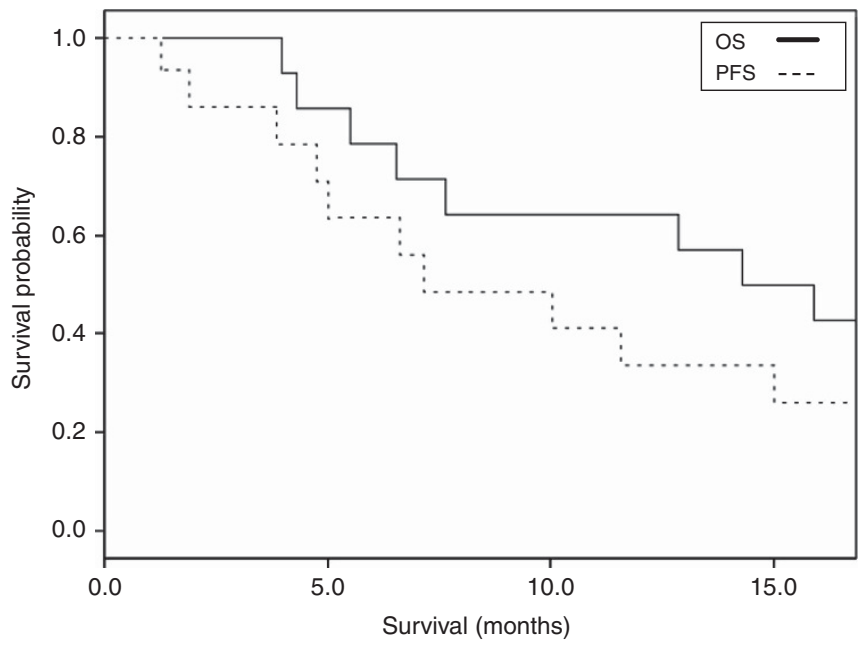

Figure 1. Kaplan-Meier curves showing overall (OS) and progressionfree survival (PFS).

response was observed in nine patients (64\%). Interestingly, nine patients $(64 \%)$ in the cohort had shown disease progression on their previous treatment protocols and six of these (66\%) then went on to have a response to the gemcitabine, carboplatin and bevacizumab; one individual (7\%) developed brain metastases following commencement of gemcitabine, carboplatin and bevacizumab.

To the best of our knowledge, this is the first-line treatment with gemcitabine, carboplatin and bevacizumab described in metastatic breast cancer. It is difficult to make comments on efficacy from a single arm study, but the PFS appears to be comparable with other regimens in late-stage disease.

Bevacizumab combined with paclitaxel has been shown to improve PFS when compared with first-line taxane therapy alone in individuals with HER-2-negative metastatic breast cancer (Miller et al, 2007), although its benefits in combination with other cytotoxics has been questioned particularly when costeffectiveness is considered (D'Agostino, 2011). In one study in which bevacizumab was administered as a second-line treatment protocol in triple-negative breast cancer, the median PFS was 6.0 months (Brufsky et al, 2012), similar numerically to the results observed here, though any comparison between studies should be interpreted with caution. In our cohort a further two women were treated with gemcitabine, cisplatin and bevacizumab, and data were similar to those observed here, but we did not include these in the primary analysis.

In summary, gemcitabine, carboplatin and bevacizumab is a well-tolerated regime that may provide an attractive treatment option in patients whose disease is continuing to progress despite ongoing interventions, who are not eligible for clinical trials. As suggested by Miles et al (2013), plasma VEGF-A and VEGFR-2 levels may be potential predictive biomarkers in this patients too.

\section{CONFLICT OF INTEREST}

The authors declare no conflict of interest.

\section{REFERENCES}

Brufsky A, Valero V, Tiangco B, Dakhil S, Brize A, Rugo HS, Rivera R, Duenne A, Bousfoul N, Yardley DA (2012) Second-line bevacizumabcontaining therapy in patients with triple-negative breast cancer: subgroup analysis of the RIBBON-2 trial. Breast Cancer Res Treat 133: 1067-1075.

Brufsky AM, Hurvitz S, Perez E, Swamy R, Valero V, O’Neill V, Rugo HS (2011) RIBBON-2: a randomized, double-blind, placebo-controlled, phase III trial evaluating the efficacy and safety of bevacizumab in combination with chemotherapy for second-line treatment of human epidermal growth factor receptor 2-negative metastatic breast cancer. J Clin Oncol 29: 4286-4293.

D’Agostino Sr RB (2011) Changing end points in breast-cancer drug approval-the Avastin story. N Engl J Med 365: e2.

Foulkes WD, Smith IE, Reis-Filho JS (2010) Triple-negative breast cancer. N Engl J Med 363: 1938-1948.

Hamilton EP, Blackwell KL (2011) Safety of bevacizumab in patients with metastatic breast cancer. Oncology 80: 314-325.

Heinemann V, Stemmler HJ, Wohlrab A, Bosse D, Losem C, Kahlert S, Rauthe G (2006) High efficacy of gemcitabine and cisplatin in patients with predominantly anthracycline- and taxane-pretreated metastatic breast cancer. Cancer Chemother Pharmacol 57: 640-646.

Jones A, Ellis P (2011) Potential withdrawal of bevacizumab for the treatment of breast cancer. Br Med J 343: d4946.

Kesikli A, Kilickap S (2012) Paclitaxel, not bevacizumab, was primarily responsible for the favorable effects seen in the RIBBON-2 trial. J Clin Oncol 30: 461; author reply 461-462.

Kitchen D, Hughes B, Gill I, O’Brien M, Rumbles S, Ellis P, Harper P, Stebbing J, Rohatgi N (2012) The relationship between vitamin D and chemotherapy-induced toxicity-a pilot study. Br J Cancer 107: 158-160.

Maisano R, Zavettieri M, Azzarello D, Raffaele M, Maisano M, Bottari M, Nardi M (2011) Carboplatin and gemcitabine combination in metastatic triple-negative anthracycline- and taxane-pretreated breast cancer patients: a phase II study. J Chemother 23: 40-43.

Miles DW, de Haas SL, Dirix LY, Romieu G, Chan A, Pivot X, Tomczak P, Provencher L, Cortes J, Delmar PR, Scherer SJ (2013) Biomarker results from the AVADO phase 3 trial of first-line bevacizumab plus docetaxel for HER2-negative metastatic breast cancer. Br J Cancer 108: 1052-1060.

Miller K, Wang M, Gralow J, Dickler M, Cobleigh M, Perez EA, Shenkier T, Cella D, Davidson NE (2007) Paclitaxel plus bevacizumab versus paclitaxel alone for metastatic breast cancer. N Engl J Med 357: 2666-2676.

O'Shaughnessy JA (2012) Breast cancer in focus: treatment options for triple-negative metastatic breast cancer. Clin Adv Hematol Oncol 10: $43-45$.

O'Shaughnessy JA, Brufsky AM (2008) RiBBON 1 and RiBBON 2: phase III trials of bevacizumab with standard chemotherapy for metastatic breast cancer. Clin Breast Cancer 8: 370-373. 
Perez EA (2004) Gemcitabine and platinum combinations in patients with breast cancer previously treated with anthracyclines and/or taxanes. Clin Breast Cancer 4(Suppl 3): S113-S116.

Sanchez-Escribano Morcuende R, Ales-Martinez JE, Aramburo Gonzalez PM (2007) Low dose gemcitabine plus cisplatin in a weekly-based regimen as salvage therapy for relapsed breast cancer after taxane-anthracyclinecontaining regimens. Clin Transl Oncol 9: 459-464.
Stebbing J, Ellis P, Tutt A (2010) PARP inhibitors in BRCA1-/BRCA2associated and triple-negative breast cancers. Future Oncol 6: 485-486.

(c) (i) (2)(2) This work is licensed under the Creative Commons (c) ${ }_{\mathrm{BY}} \mathrm{NC}_{\mathrm{SA}}$ Attribution-NonCommercial-Share Alike 3.0 Unported License. To view a copy of this license, visit http://creativecommons. org/licenses/by-nc-sa/3.0/ 